\title{
L-P Perturbation Solution of Nonlinear Free Vibration of Prestressed Orthotropic Membrane in Large Amplitude
}

\author{
Liu Chang-jiang, ${ }^{1}$ Zheng Zhou-lian, ${ }^{1,2}$ He Xiao-ting, ${ }^{1}$ \\ Sun Jun-yi, ${ }^{1}$ Song Wei-ju, ${ }^{1}$ Xu Yun-ping, ${ }^{1}$ and Long Jun ${ }^{1}$ \\ ${ }^{1}$ College of Civil Engineering, Chongqing University, Chongqing 400045, China \\ ${ }^{2}$ Key Laboratory of the Three Gorges Reservoir Region's Eco-Environment, \\ Ministry of Education, Chongqing 400045, China \\ Correspondence should be addressed to Liu Chang-jiang, changjiangliucd@126.com
}

Received 25 May 2010; Accepted 30 September 2010

Academic Editor: Jaromir Horacek

Copyright (C) 2010 Liu Chang-jiang et al. This is an open access article distributed under the Creative Commons Attribution License, which permits unrestricted use, distribution, and reproduction in any medium, provided the original work is properly cited.

This paper reviewed the research on the nonlinear free vibration of pre-stressed orthotropic membrane, which is commonly applied in building membrane structures. We applied the L$\mathrm{P}$ perturbation method to solve the governing equations of large amplitude nonlinear free vibration of rectangular orthotropic membranes and obtained a simple approximate analytical solution of the frequency and displacement function of large amplitude nonlinear free vibration of rectangular membrane with four edges simply supported. By giving computational examples, we compared and analyzed the frequency results. In addition, vibration mode of the membrane and displacement and time curve of each feature point on the membrane surface were analyzed in the computational example. Results obtained from this paper provide a simple and convenient method to calculate the frequency and lateral displacement of nonlinear free vibration of rectangular orthotropic membranes in large amplitude. Meanwhile, the results provide some theoretical basis for solving the response of membrane structures under dynamic loads and provide some computational basis for the vibration control and dynamic design of building membrane structures.

\section{Introduction}

The orthotropic membrane materials are mainly applied in building membrane structures. The membrane structure is a thin flexible structure, so it is easy to engender vibration and relaxation deformation, thus results in engineering accident. Therefore, it is quite necessary to study the vibration characteristic of membrane structure to provide some computational basis for the design of membrane structure to ensure the safety of membrane structure. 
The amplitude of the membrane is much larger than its thickness in vibration process, so the geometric nonlinearity caused by large amplitude must be considered. At present, there are some reports about the nonlinear vibration problem of membranes. Shin et al. [1] investigated the geometric nonlinear dynamic characteristics of the out-of-plane vibration of an axially moving membrane by using Hamilton's principle and Galerkin's method. The results showed that the translating speed, boundary conditions and aspect ratio of the membrane have effects on the natural frequencies, mode shapes and stability for the out-of-plane vibration of the moving membrane. Zhang and Shan [2] studied initial equilibrium shape and free vibration properties pretensioned membrane structures by applying relaxation method. Example analysis demonstrated that the method is a simple, practical and accurate geometric nonlinear method to study the tensioned cladding-network with big span. Li et al. [3] presented NBA-UM (Nonlinear Buckling Analysis-Update Matrix) method to analyze the vibration behaviors of the wrinkled membrane. The results reveal that the wrinkles have great effects on the free vibration characteristics of wrinkled membrane. Pan and Gu [4] studied the effects of membrane's prestrain, size, elastic ratio, density, relative amplitude and dead load of square tensioned membrane to the structure's nonlinearity and deduced the free oscillating system's equivalent fundamental frequency. Reutskiy [5] adopted a new numerical method to study nonlinear vibration of arbitrarily shaped membranes. The method is based on mathematical modeling of physical response of a system that was excited over a range of frequencies. Formosa [6] presented a work devoted to the study of the operation of a miniaturized membrane Stirling engine. The nonlinearities of large amplitude vibration of Stirling engine were concerned, and the stability analysis to predict the starting of the engine and the instability problem that leads to the steady-state behavior were displayed. Gonçalves et al. [7] presented a detailed analysis of the geometrically and materially nonlinear vibration response of a prestretched hyperelastic membrane subjected to finite deformations and a time-varying lateral pressure by using Galerkin's method and nonlinear finite element method. The results showed the strong influence of the stretching ratio on the linear and nonlinear oscillations of the membrane. The research objects of these above researches are all isotropic membrane material. There are a few reports about the nonlinear vibration of orthotropic membrane material. Zheng Zhou-lian et al. [8] studied the geometric nonlinear vibration of orthotropic membranes. They established the governing equations by applying Von Kármán's large deflection theory and D'Alembert's principle of membrane, and applied Galerkin's method and power series expansion method to solve the governing equations. They obtained the power series solution of nonlinear vibration frequency of rectangular membrane with four edges xed, but the power series formula is so complex that it is not very convenient for calculating and the displacement function of the vibration membrane was not be solved.

In this paper, we apply the L-P perturbation method to solve the nonlinear free vibration problem of orthotropic membranes in large amplitude. The L-P perturbation is one kind of singular perturbation method, which was originated by Lindstedt and Poincaré. The central idea of this method is to construct a progressive analytical expression to replace the accurate solution of the definite problem of differential equation according to some artificial specific steps. The applications of this method in many actual engineering problems proved that this method is an effective mathematical method to solve the approximate analytical solution of nonlinear differential equation [9-19].

Through using the L-P perturbation method to solve the governing equations of large amplitude nonlinear free vibration of rectangular orthotropic membranes in this paper, we obtained an approximate analytical frequency solution and displacement function of the 


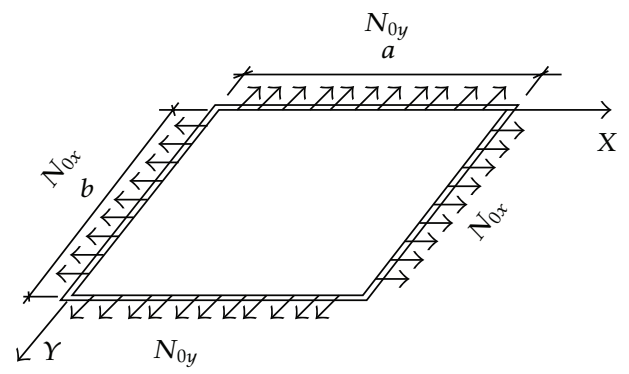

Figure 1: Rectangle membrane with four edges simply supported.

nonlinear free vibration of prestressed orthotropic rectangular membrane with four edges simply supported. Results obtained from this paper provide a simple and convenient new method to calculate the frequency and lateral displacement of large amplitude nonlinear free vibration of rectangular orthotropic membranes. Meanwhile, the results provide some theoretical basis for solving the response of membrane structures under dynamic loads, and provide some computational basis for the vibration control and dynamic design of building membrane structures.

\section{Governing Equations and Boundary Conditions}

The membrane material studied is orthotropic. Its two orthogonal directions are the two principal fiber directions, and the material characteristics of the two principal fiber directions are different. Assume that the rectangular membrane studied is simply supported on its four edges. The two principal fiber directions are $x$ and $y$, respectively. $a$ and $b$ denote the length of $x$ and $y$ direction, respectively; $N_{0 x}$ and $N_{0 y}$ denote initial tension in $x$ and $y$, respectively, as shown in Figure 1.

In the process of vibration, although the amplitude is much smaller than the boundary size of membrane, it is not much smaller than its thickness. Therefore, the geometric nonlinearity must be considered in the process of membrane vibration.

According to the Von Kármán's large deflection theory and D'Alembert's principle, the vibration partial differential equation and consistency equation of orthotropic membrane [8] are

$$
\begin{gathered}
\rho \frac{\partial^{2} w}{\partial t^{2}}-\left(N_{x}+N_{0 x}\right) \frac{\partial^{2} w}{\partial x^{2}}-\left(N_{y}+N_{0 y}\right) \frac{\partial^{2} w}{\partial y^{2}}=0 \\
\frac{1}{E_{1} h} \frac{\partial^{2} N_{x}}{\partial y^{2}}-\frac{\mu_{2}}{E_{2} h} \frac{\partial^{2} N_{y}}{\partial y^{2}}-\frac{\mu_{1}}{E_{1} h} \frac{\partial^{2} N_{x}}{\partial x^{2}}+\frac{1}{E_{2} h} \frac{\partial^{2} N_{y}}{\partial x^{2}}-\frac{1}{G h} \frac{\partial^{2} N_{x y}}{\partial x \partial y}=\left(\frac{\partial^{2} w}{\partial x \partial y}\right)^{2}-\frac{\partial^{2} w}{\partial x^{2}} \frac{\partial^{2} w}{\partial y^{2}}
\end{gathered}
$$

where $\rho$ denotes aerial density of membrane. $N_{x}$ and $N_{y}$ denote additional tension in $x$ and $y$ direction, respectively. $N_{0 x}$ and $N_{0 y}$ denote initial tension in $x$ and $y$ direction, respectively. $N_{x y}$ denotes shear force. $w$ denotes deflection. $w(x, y, t) ; h$ denotes membrane's thickness. $E_{1}$ and $E_{2}$ denote Young's modulus in $x$ and $y$ direction, respectively. $G$ denotes shearing modulus. $\mu_{1}$ and $\mu_{2}$ denote Poisson's ratio in $x$ and $y$ direction, respectively. 
While the membrane is in vibration, the effect of shearing stress is so small that we may take $N_{x y}=0$, Introduce the stress function

$$
\begin{array}{ll}
N_{x}=h \frac{\partial^{2} \varphi}{\partial y^{2}}, & N_{0 x}=h \cdot \sigma_{0 x}, \\
N_{y}=h \frac{\partial^{2} \varphi}{\partial x^{2}}, & N_{0 y}=h \cdot \sigma_{0 y} .
\end{array}
$$

Equation (2.1) can be simplified as follows:

$$
\begin{gathered}
\frac{\rho}{h} \frac{\partial^{2} w}{\partial t^{2}}-\left(\sigma_{0 x}+\frac{\partial^{2} \varphi}{\partial y^{2}}\right) \frac{\partial^{2} w}{\partial x^{2}}-\left(\sigma_{0 y}+\frac{\partial^{2} \varphi}{\partial x^{2}}\right) \frac{\partial^{2} w}{\partial y^{2}}=0, \\
\frac{1}{E_{1}} \frac{\partial^{4} \varphi}{\partial y^{4}}+\frac{1}{E_{2}} \frac{\partial^{4} \varphi}{\partial x^{4}}=\left(\frac{\partial^{2} w}{\partial x \partial y}\right)^{2}-\frac{\partial^{2} w}{\partial x^{2}} \frac{\partial^{2} w}{\partial y^{2}} .
\end{gathered}
$$

where $\varphi$ denotes stress function $\varphi(x, y, t), \sigma_{0 x}$ and $\sigma_{0 y}$ denote initial tensile stress in $x$ and $y$ direction, respectively.

The corresponding boundary conditions are as follows:

$$
\begin{aligned}
w(0, y, t)=0, & \frac{\partial^{2} w}{\partial x^{2}}(0, y, t)=0, \\
w(a, y, t)=0, & \frac{\partial^{2} w}{\partial x^{2}}(a, y, t)=0, \\
w(x, 0, t)=0, & \frac{\partial^{2} w}{\partial y^{2}}(x, 0, t)=0, \\
w(x, b, t)=0, & \frac{\partial^{2} w}{\partial y^{2}}(x, b, t)=0 .
\end{aligned}
$$

\section{Simplification of Governing Equations}

Functions that satisfy the boundary conditions (2.5) are expressed as follows:

$$
\begin{gathered}
w(x, y, t)=W(x, y) T(t), \\
\varphi(x, y, t)=\phi(x, y) T^{2}(t),
\end{gathered}
$$

where $W(x, y)$ is the given mode shape function, $\phi(x, y)$ and $T(t)$ are the unknown functions. Assume that the mode shape function is as follows:

$$
W(x, y)=\sin \frac{m \pi x}{a} \sin \frac{n \pi y}{b},
$$


where $m$ and $n$ are integer, and denote the sine half-wave number in $x$ and $y$, respectively. Equation (3.3) satisfies the boundary conditions automatically.

Substituting (3.1) and (3.2) into (2.4) yields

$$
\frac{1}{E_{1}} \frac{\partial^{4} \phi}{\partial y^{4}}+\frac{1}{E_{2}} \frac{\partial^{4} \phi}{\partial x^{4}}=\left(\frac{\partial^{2} W}{\partial x \partial y}\right)^{2}-\frac{\partial^{2} W}{\partial x^{2}} \frac{\partial^{2} W}{\partial y^{2}}
$$

Substituting (3.3) into (3.4) yields

$$
\frac{1}{E_{1}} \frac{\partial^{4} \phi}{\partial y^{4}}+\frac{1}{E_{2}} \frac{\partial^{4} \phi}{\partial x^{4}}=\frac{m^{2} n^{2} \pi^{4}}{2 a^{2} b^{2}}\left(\cos \frac{2 m \pi x}{a}+\cos \frac{2 n \pi y}{b}\right)
$$

Assume the solution of (3.5) is:

$$
\phi(x, y)=\alpha \cdot \cos \frac{2 m \pi x}{a}+\beta \cdot \cos \frac{2 n \pi y}{b} .
$$

Substituting (3.6) into (3.5) yields

$$
\alpha=\frac{E_{2} n^{2} a^{2}}{32 m^{2} b^{2}}, \quad \beta=\frac{E_{1} m^{2} b^{2}}{32 n^{2} a^{2}} .
$$

Substituting (3.1) and (3.2) into (2.3), according to the Galerkin method yields

$$
\begin{gathered}
\iint_{S}\left(\frac{\rho}{h} \frac{\partial^{2} w}{\partial t^{2}}-\left(\sigma_{0 x}+\frac{\partial^{2} \varphi}{\partial y^{2}}\right) \frac{\partial^{2} w}{\partial x^{2}}-\left(\sigma_{0 y}+\frac{\partial^{2} \varphi}{\partial x^{2}}\right) \frac{\partial^{2} w}{\partial y^{2}}\right) W(x, y) d s \\
=\iint_{S}\left[\frac{\rho}{h} W \frac{\partial^{2} T(t)}{\partial t^{2}}-\left(\sigma_{0 x} \cdot \frac{\partial^{2} W}{\partial x^{2}}+\sigma_{0 y} \cdot \frac{\partial^{2} W}{\partial y^{2}}\right) T(t)\right. \\
\left.-\left(\frac{\partial^{2} \phi}{\partial y^{2}} \frac{\partial^{2} W}{\partial x^{2}}+\frac{\partial^{2} \phi}{\partial x^{2}} \frac{\partial^{2} W}{\partial y^{2}}\right) T^{3}(t)\right] W(x, y) d s=0 .
\end{gathered}
$$

Obviously, (3.8) is a nonlinear differential equation with respect to $T(t)$. It can be expressed as follows:

$$
A \cdot \frac{d^{2} T(t)}{d t^{2}}-B \cdot T(t)-C \cdot T^{3}(t)=0
$$


where

$$
\begin{gathered}
A=\iint_{S} \frac{\rho}{h} W^{2} d s=\iint_{S} \frac{\rho}{h} \sin ^{2} \frac{m \pi x}{a} \sin ^{2} \frac{n \pi y}{b} d s=\frac{\rho a b}{4 h} \\
B=\iint_{S}\left(\sigma_{0 x} \cdot \frac{\partial^{2} W}{\partial x^{2}}+\sigma_{0 y} \cdot \frac{\partial^{2} W}{\partial y^{2}}\right) W d s=-\frac{\pi^{2} a b}{4}\left(\frac{m^{2}}{a^{2}} \sigma_{0 x}+\frac{n^{2}}{b^{2}} \sigma_{0 y}\right), \\
C=\iint_{S}\left(\frac{\partial^{2} \phi}{\partial y^{2}} \frac{\partial^{2} W}{\partial x^{2}}+\frac{\partial^{2} \phi}{\partial x^{2}} \frac{\partial^{2} W}{\partial y^{2}}\right) W d s=-\frac{a b \pi^{4}}{64}\left(\frac{E_{1} m^{4}}{a^{4}}+\frac{E_{2} n^{4}}{b^{4}}\right) .
\end{gathered}
$$

Substituting the value of $A, B$ and $C$ into (3.9) yields

$$
\frac{d^{2} T(t)}{d t^{2}}+\frac{h \pi^{2}}{\rho}\left(\frac{m^{2}}{a^{2}} \sigma_{0 x}+\frac{n^{2}}{b^{2}} \sigma_{0 y}\right) T(t)+\frac{h \pi^{4}}{16 \rho}\left(\frac{E_{1} m^{4}}{a^{4}}+\frac{E_{2} n^{4}}{b^{4}}\right) T^{3}(t)=0
$$

\section{The L-P Perturbation Solution of Governing Equations}

Apply the L-P perturbation method to obtain the approximate analytical solution that satisfy (3.11). Assuming that the perturbation parameter is $\varepsilon=\left(h^{2} / a b\right) \ll 1$, (3.11) can be simplified as follows:

$$
\frac{d^{2} T(t)}{d t^{2}}+\omega_{0}^{2}\left(T(t)+\varepsilon \cdot \alpha_{1} \cdot T^{3}(t)\right)=0
$$

where $\omega_{0}^{2}=\left(h \pi^{2} / \rho\right)\left(\left(m^{2} / a^{2}\right) \sigma_{0 x}+\left(n^{2} / b^{2}\right) \sigma_{0 y}\right), \alpha_{1}=\left(\pi^{4} a b / 16 \rho h \omega_{0}^{2}\right)\left(\left(E_{1} m^{4} / a^{4}\right)+\left(E_{2} n^{4} / b^{4}\right)\right)$.

Introducing a new variable: $\tau=\omega \cdot t$, then (4.1) can be expressed as

$$
\omega^{2} \frac{d^{2} T(\tau)}{d \tau^{2}}+\omega_{0} T(\tau)=-\varepsilon \alpha_{1} \omega_{0}^{2} T^{3}(\tau)
$$

Spread $\omega$ and $T(\tau)$ as a power series with respect to $\varepsilon$ :

$$
\begin{gathered}
T(\tau)=T_{0}(\tau)+\varepsilon T_{1}(\tau)+\varepsilon^{2} T_{2}(\tau)+\cdots+\varepsilon^{n} T_{n}(\tau)+O\left(\varepsilon^{n+1}\right), \\
\omega=\omega_{0}+\varepsilon \omega_{1}+\varepsilon^{2} \omega_{2}+\cdots+\varepsilon^{n} \omega_{n}+O\left(\varepsilon^{n}\right) .
\end{gathered}
$$

Substituting (4.3) into (4.2) yields

$$
\begin{gathered}
\left(\omega_{0}+\varepsilon \omega_{1}+\varepsilon^{2} \omega_{2}+\cdots\right)^{2}\left(\ddot{T}_{0}(\tau)+\varepsilon \ddot{T}_{1}(\tau)+\varepsilon^{2} \ddot{T}_{2}(\tau)+\cdots\right) \\
+\omega_{0}^{2}\left(T_{0}(\tau)+\varepsilon T_{1}(\tau)+\varepsilon^{2} T_{2}(\tau)+\cdots\right) \\
=-\varepsilon \alpha_{1} \omega_{0}^{2}\left(T_{0}(\tau)+\varepsilon T_{1}(\tau)+\varepsilon^{2} T_{2}(\tau)+\cdots\right)^{3} .
\end{gathered}
$$

In (4.4), $\ddot{T}(\tau)=d^{2} T(\tau) / d \tau^{2}, \dot{T}(\tau)=d T(\tau) / d \tau$. 
Spread (4.4), and compare the coefficient of each power of $\varepsilon$ yields

(1) $\varepsilon^{0}$ :

$$
\ddot{T}_{0}(\tau)+T_{0}(\tau)=0
$$

The general solution of (4.5) is

$$
T_{0}(\tau)=C_{1} \cdot \cos \tau+C_{2} \cdot \sin \tau
$$

Assume that the initial condition is:

$$
\begin{gathered}
T(0)=T_{0}(0)+\varepsilon T_{1}(0)+\varepsilon^{2} T_{2}(0)+\cdots=a_{0}, \\
\dot{T}(0)=\dot{T}_{0}(0)+\varepsilon \dot{T}_{1}(0)+\varepsilon^{2} \dot{T}_{2}(0)+\cdots=0 .
\end{gathered}
$$

According to (4.7), we can obtain: $T_{0}(0)=a_{0}, \dot{T}_{0}(0)=0$, substituting them into (4.6) yields

$$
T_{0}(\tau)=a_{0} \cdot \cos \tau
$$

(2) $\varepsilon^{1}$ :

$$
2 \omega_{0} \omega_{1} \ddot{T}_{0}(\tau)+\omega_{0}^{2} \ddot{T}_{1}(\tau)+\omega_{0}^{2} T_{1}(\tau)=-\alpha_{1} \omega_{0}^{2} T_{0}^{3}(\tau)
$$

Substituting (4.8) into (4.9) yields

$$
\ddot{T}_{1}(\tau)+T_{1}(\tau)=-\frac{3}{4} \alpha_{1} a_{0}^{3} \cos \tau-\frac{1}{4} \alpha_{1} a_{0}^{3} \cos 3 \tau+2 \frac{\omega_{1}}{\omega_{0}} a_{0} \cos \tau .
$$

A homogeneous general solution of (4.10) is $g=\cos \tau$, The inhomogeneous term of (4.10) is

$$
f=-\frac{3}{4} \alpha_{1} a_{0}^{3} \cos \tau-\frac{1}{4} \alpha_{1} a_{0}^{3} \cos 3 \tau+2 \frac{\omega_{1}}{\omega_{0}} a_{0} \cos \tau .
$$

In order to make the general solution of (4.10) not contain secular terms, we must orthogonalize g and $f$, namely $\langle g, f\rangle=0$ yields

$$
2 \frac{\omega_{1}}{\omega_{0}} a_{0}-\frac{3}{4} \alpha_{1} a_{0}^{3}=0 .
$$

According to (4.12) yields

$$
\omega_{1}=\frac{3 \omega_{0} \alpha_{1} a_{0}^{2}}{8} .
$$


Substituting (4.13) into (4.10) yields

$$
\ddot{T}_{1}(\tau)+T_{1}(\tau)=-\frac{1}{4} \alpha_{1} a_{0}^{3} \cos 3 \tau
$$

The general solution of $T_{1}(\tau)$ is:

$$
T_{1}(\tau)=C_{3} \cdot \cos \tau+C_{4} \cdot \sin \tau+\frac{\alpha_{1} a_{0}^{3}}{32} \cos 3 \tau
$$

According to (4.7), we can obtain: $T_{1}(0)=0, \dot{T}_{1}(0)=0$ substituting them into (4.15) yields

$$
T_{1}(\tau)=\frac{\alpha_{1} a_{0}^{3}}{32}(\cos 3 \tau-\cos \tau)
$$

Substituting (4.8), (4.16), and (4.13) into (4.3) yields

$$
\begin{gathered}
T(\tau)=a_{0} \cdot \cos \tau+\frac{\alpha_{1} a_{0}^{3} \varepsilon}{32}(\cos 3 \tau-\cos \tau)+O\left(\varepsilon^{2}\right), \\
\omega=\omega_{0}+\frac{3 \omega_{0} \alpha_{1} a_{0}^{2} \varepsilon}{8}+O\left(\varepsilon^{2}\right) .
\end{gathered}
$$

Substituting (4.18) into $\tau=\omega t$ yields

$$
\tau=\left(\omega_{0}+\frac{3 \omega_{0} \alpha_{1} a_{0}^{2} \varepsilon}{8}+O\left(\varepsilon^{2}\right)\right) \cdot t .
$$

Substituting (4.19) into (4.17) yields

$$
\begin{aligned}
T(t)= & a_{0} \cdot \cos \left(1+\frac{3 \alpha_{1} a_{0}^{2} \varepsilon}{8}\right) \omega_{0} t \\
& +\frac{\alpha_{1} a_{0}^{3} \varepsilon}{32}\left(\cos 3\left(1+\frac{3 \alpha_{1} a_{0}^{2} \varepsilon}{8}\right) \omega_{0} t-\cos \left(1+\frac{3 \alpha_{1} a_{0}^{2} \varepsilon}{8}\right) \omega_{0} t\right) .
\end{aligned}
$$

Equation (4.20) is the approximate analytical solution of (3.11). According to (4.18), the approximate analytical expression of the frequency is

$$
\omega=\omega_{0}\left(1+\frac{3 \alpha_{1} a_{0}^{2} \varepsilon}{8}\right)
$$


In [8], the power series frequency solution of the vibration of rectangular orthotropic membranes in large deflection is

$$
\omega=\frac{2 \pi}{Z}=\frac{\sqrt{M+(N / 2) a_{0}^{2}}}{\sum_{n=0}^{\infty}(-1)^{p}((2 p-1) ! ! /(2 p) ! !)^{2}\left(N a_{0}^{2} /\left(2 M+N a_{0}^{2}\right)\right)^{p}},
$$

where $M=\left(h \pi^{2} / \rho\right)\left(\left(m^{2} / a^{2}\right) \sigma_{0 x}+\left(n^{2} / b^{2}\right) \sigma_{0 y}\right), N=\left(\pi^{4} h / 16 \rho\right)\left(E_{1} m^{4} / a^{4}+E_{2} n^{4} / b^{4}\right)$ and $a_{0}$ is the amplitude of the membrane, $p=0,1,2,3, \ldots$.

In (4.21) and (4.22), let $a_{0} \rightarrow 0,(4.21)$ and (4.22) can be transformed into the frequency formula of the vibration of rectangular orthotropic membranes in small deflection:

$$
\omega=\omega_{0}=\pi \sqrt{\frac{h}{\rho}} \cdot \sqrt{\frac{m^{2}}{a^{2}} \sigma_{0 x}+\frac{n^{2}}{b^{2}} \sigma_{0 y}} .
$$

This proves that the frequency formula of this paper is qualitatively consistent with the result in paper [8]. In the computational example, the results calculated according to (4.21), (4.22) and (4.23) will be compared and analyzed.

Substituting (3.3) and (4.20) into (3.1), we can obtain the displacement function of nonlinear free vibration of rectangular orthotropic membranes in large amplitude:

$$
\begin{aligned}
w(x, y, t)= & \sum_{m=1}^{\infty} \sum_{n=1}^{\infty} \sin \frac{m \pi x}{a} \sin \frac{n \pi y}{b} \\
& \times\left(a_{0} \cos \left(1+\frac{3 \alpha_{1} a_{0}^{2} \varepsilon}{8}\right) \omega_{0} t\right. \\
& \left.+\frac{\alpha_{1} a_{0}^{3} \varepsilon}{32}\left(\cos 3\left(1+\frac{3 \alpha_{1} a_{0}^{2} \varepsilon}{8}\right) \omega_{0} t-\cos \left(1+\frac{3 \alpha_{1} a_{0}^{2} \varepsilon}{8}\right) \omega_{0} t\right)\right)
\end{aligned}
$$

We can obtain the lateral displacement of any point on the membrane surface according to (4.24), and analyze the vibration modes and displacement time histories of each point on the membrane surface.

\section{Computational Example and Discussion}

Take the membrane material commonly applied in project as an example. The Young's modulus in $x$ and $y$ are $E_{1}=1.4 \times 10^{6} \mathrm{KN} / \mathrm{m}^{2}$ and $E_{2}=0.9 \times 10^{6} \mathrm{KN} / \mathrm{m}^{2}$, respectively; the aerial density of membranes is $\rho=1.7 \mathrm{~kg} / \mathrm{m}^{2}$; the membrane's thickness is $h=1.0 \mathrm{~mm}$, $a=1 \mathrm{~m}, b=1 \mathrm{~m}, \sigma_{0 x}=\sigma_{0 y}=5.0 \times 10^{3} \mathrm{KN} / \mathrm{m}^{2}$.

\subsection{Calculation of Frequency}

The frequencies of the first nine orders under different initial conditions are calculated according to (4.21), (4.22) and (4.23). The results are shown in Table 1. 


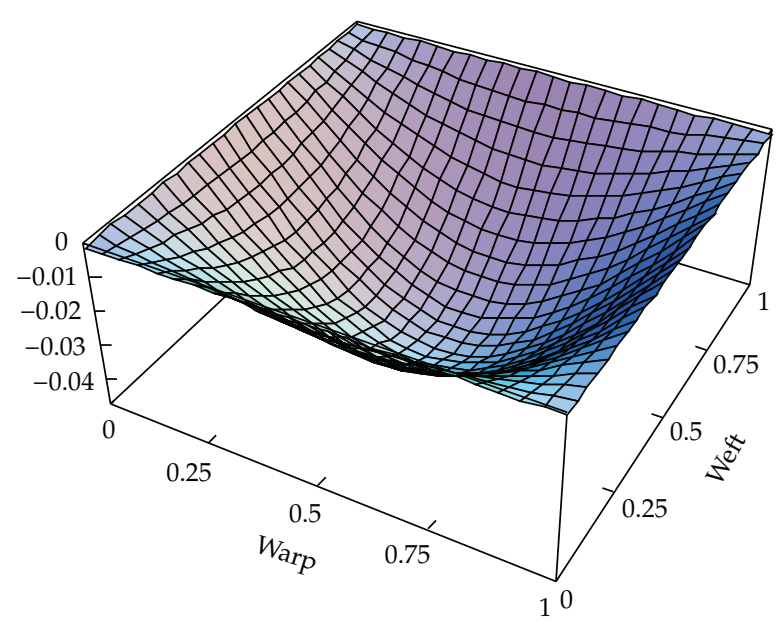

Figure 2: The first-order vibration mode.

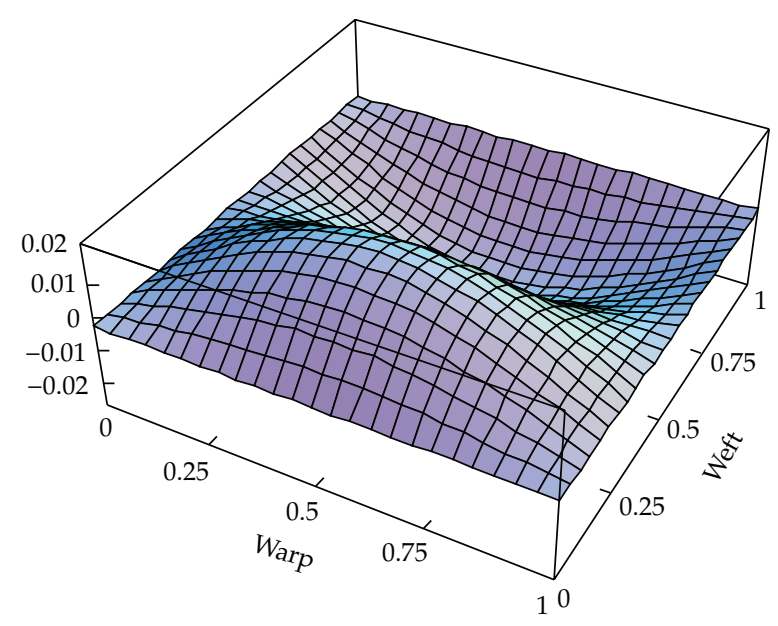

Figure 3: The second-order vibration mode.

The comparison and analysis of Table 1 are as follows.

(1) All of the frequency values calculated according to (4.21) and (4.22) enlarge with the increase of initial displacement. This is due to the fact that the inner force, lateral rigidity and elastic restoring force will increase with the increase of initial displacement of membrane, and then the membrane will vibrate more quickly. This reflects the geometric nonlinearity characteristic of the vibration of membrane in large amplitude. Meanwhile, the frequency values under the same initial displacement enlarge with the increase of orders. When the initial displacement approaches zero, namely, $a_{0} \rightarrow 0$, the frequency values calculated according to (4.21) and (4.22) are the same as the frequency values calculated according to the 


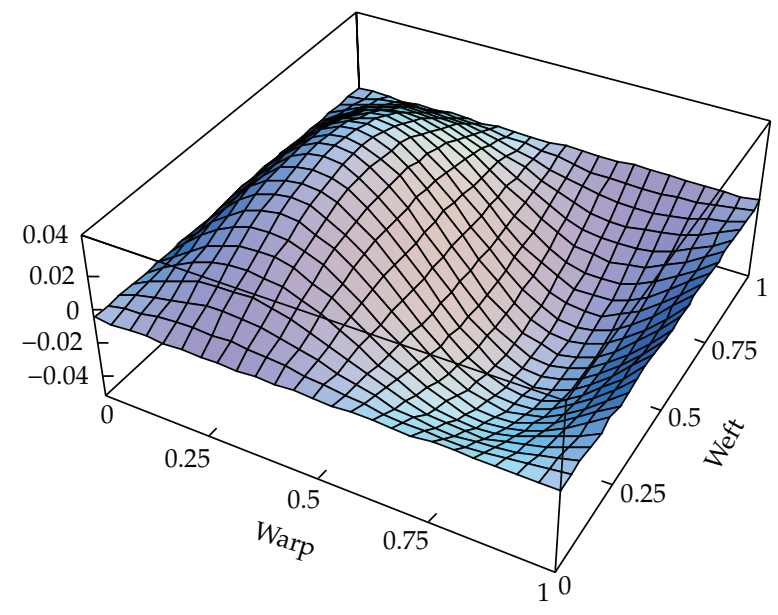

Figure 4: The third-order vibration mode.

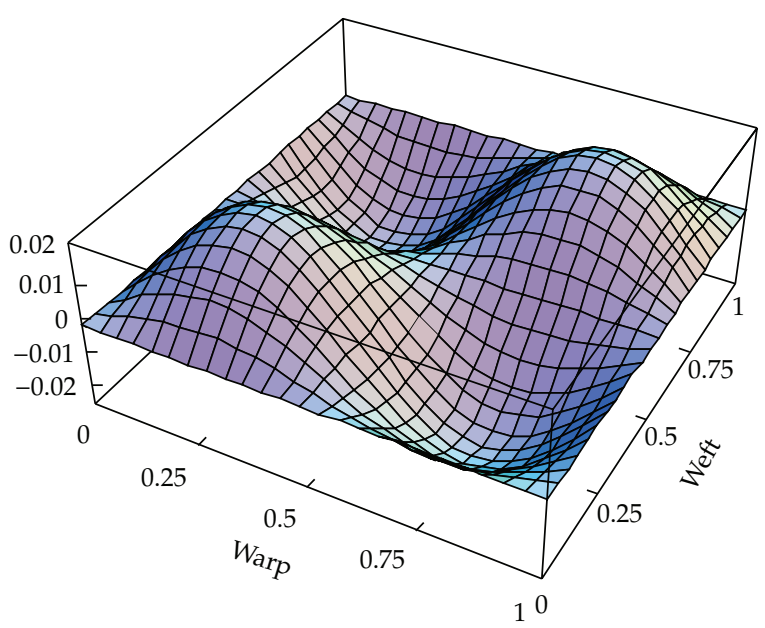

Figure 5: The fourth-order vibration mode.

small amplitude frequency formula (4.23). This further proved that the frequency formula (4.21) was qualitatively consistent with the frequency formula (4.22).

(2) The frequency values calculated according to (4.21) are slightly larger than the corresponding ones calculated according to (4.22). The relative differences become larger and larger with the increase of initial displacement; meanwhile, the relative differences also become larger and larger with the increase of order. This is because that, the geometric nonlinearity of membrane vibration will become stronger and stronger with the increase of initial displacement and order, and it will become more and more difficult for the approximate analytical solution (4.21) to reflect the accurate analytical solution. Nevertheless, when the initial displacement is small and order is low, using formula (4.21) to calculate the vibration frequency 


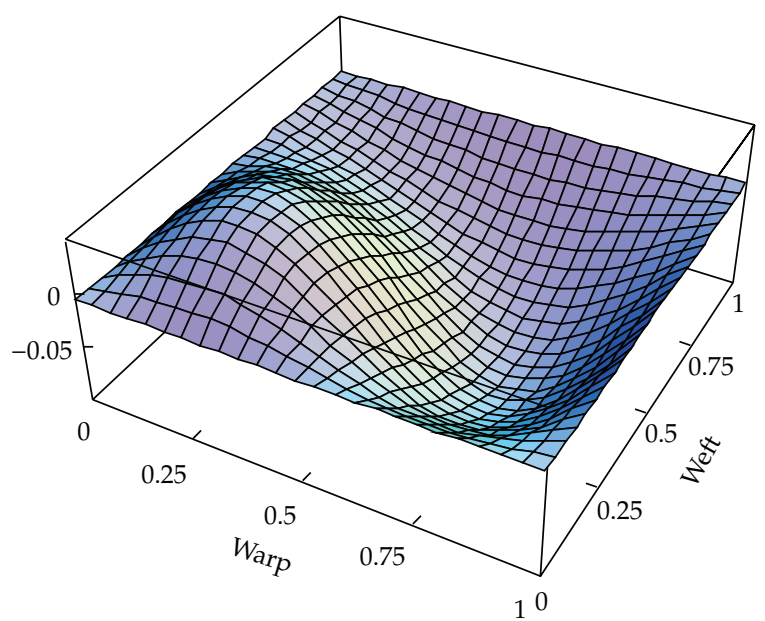

Figure 6: The superposed vibration mode of the first four orders.

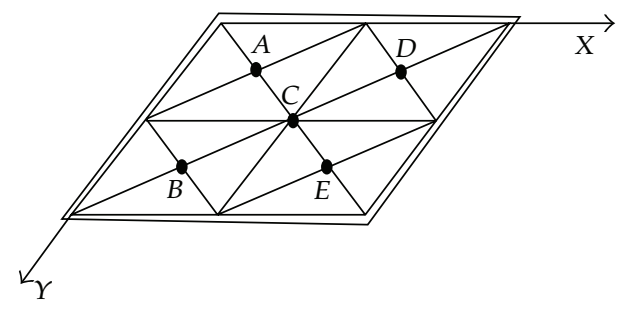

Figure 7: Feature points on membrane surface.

of membrane still satisfy the engineering precision and it is simpler and more convenient than formula (4.22).

(3) When calculating the frequency of the first-order (fundamental frequency), the results calculated according to (4.21) are very close to the results calculated according to (4.22), the largest relative difference is $6.7557 \%$. Hereby, we can infer that while $a_{0} / a \leqslant 0.1$, using formula (4.21) to calculate the fundamental frequency can satisfy the engineering precision and is simple and convenient.

(4) When calculating the frequency of the other order, we can deduce that while $a_{0} / a \leqslant 0.05$, using formula (4.21) to calculate the frequencies of the first four orders can satisfy the engineering precision; and while $a_{0} / a \leqslant 0.03$, using formula (4.21) to calculate the frequencies of the first nine orders can satisfy the engineering precision. In general, the vibration of membrane is mainly dominated by the first three orders, while $a_{0} / a \leqslant 0.5$, the largest relative difference between the frequency results of the first three orders calculated according to (4.21) and the corresponding ones calculated according to $(4.22)$ is $6.865 \%$. So, using formula (4.21) to calculate the main vibration frequency of rectangular orthotropic membranes in large deflection can satisfy the requirement of engineering precision when the initial displacement is relatively small. 


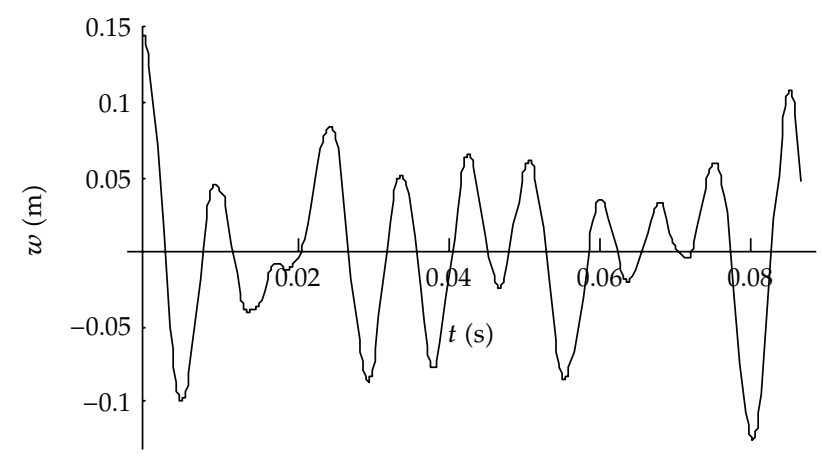

Figure 8: Displacement and time curve of $A$ point.

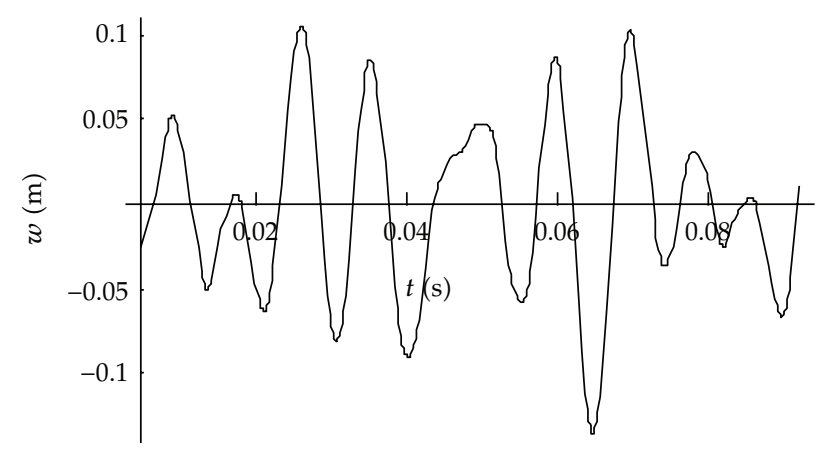

Figure 9: Displacement and time curve of $B$ point.

\subsection{Analysis of Vibration Mode}

We substituted the material and geometric parameters in computational example and the frequency values calculated according to (4.21) (while $a_{0} / a=0.05 \mathrm{~m}$ ) into (4.24) to obtain the displacement function of the vibration of the first four orders.

(1) The first-order vibration mode $(m=1, n=1)$

$$
w(x, y, t)=\left(0.05 \cos 273 t+5.54 \times 10^{-4}(\cos 819 t-\cos 273 t)\right) \sin \pi x \sin \pi y .
$$

(2) The second-order vibration mode $(m=1, n=2)$

$w(x, y, t)=\left(0.05 \cos 520 t+1.523 \times 10^{-3}(\cos 1560 t-\cos 520 t)\right) \sin \pi x \sin 2 \pi y$.

(3) The third-order vibration mode $(m=2, n=1)$

$$
w(x, y, t)=\left(0.05 \cos 586 t+2.246 \times 10^{-3}(\cos 1758 t-\cos 586 t)\right) \sin 2 \pi x \sin \pi y
$$




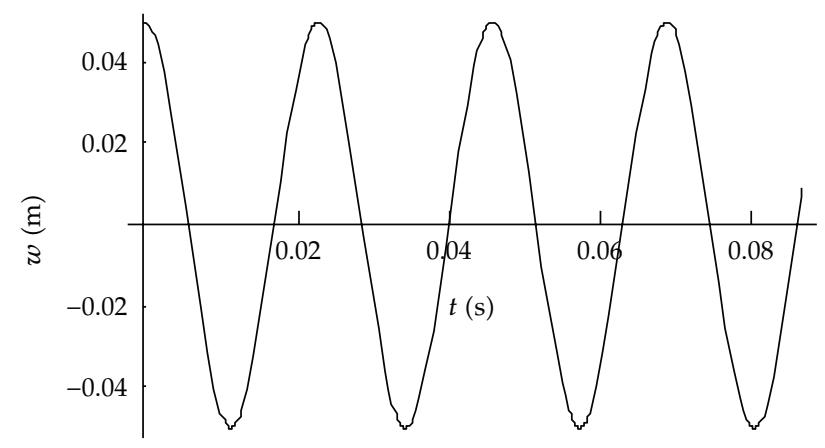

Figure 10: Displacement and time curve of $C$ point.

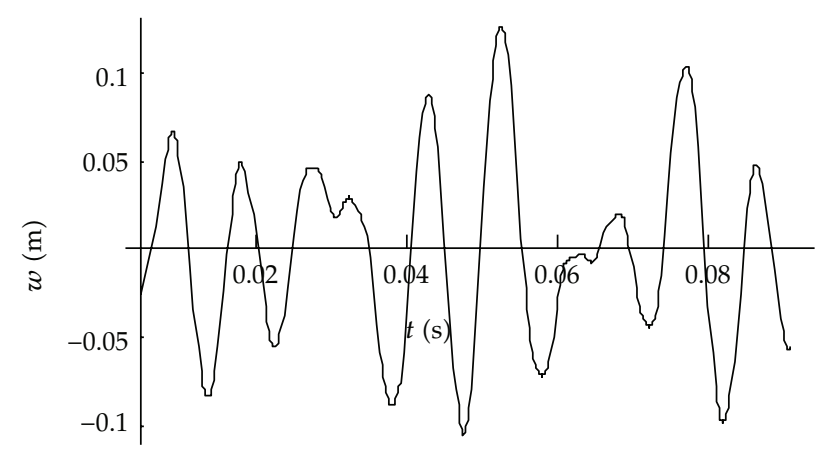

Figure 11: Displacement and time curve of $D$ point.

(4) The fourth-order vibration mode $(m=2, n=2)$

$w(x, y, t)=\left(0.05 \cos 738 t+2.217 \times 10^{-3}(\cos 2214 t-\cos 738 t)\right) \sin 2 \pi x \sin 2 \pi y$.

According to these displacement functions, we can draw the vibration mode figures of the first four orders while $t=0.01 \mathrm{~s}$. The figures are shown as Figures 2, 3, 4, and 5 .

We superposed the vibration mode of the first four orders to obtain the superposed vibration mode figure. It is shown in Figure 6.

From the result of the vibration mode analysis, we can conclude that using the deflection function (4.24) can calculate the vibration mode of each order and obtain the total superposed vibration mode of the nonlinear vibration of orthotropic membranes in large deflection conveniently.

\subsection{Analysis of Displacement Time Histories of Feature Points}

We substituted the material and geometric parameters in computational example and the frequency values calculated according to (4.21) (while $a_{0} / a=0.05 \mathrm{~m}$ ) into (4.24) to calculate the displacement time histories of the feature points on membrane surface and draw the displacement and time curves. These feature points are $A(x=0.25 \mathrm{~m}, y=0.25 \mathrm{~m})$, 


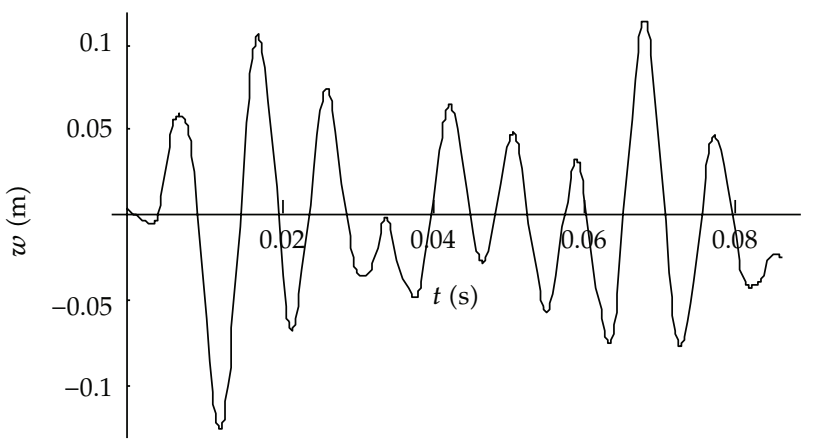

Figure 12: Displacement and time curve of $E$ point.

$B(x=0.25 \mathrm{~m}, y=0.75 \mathrm{~m}), C(x=0.5 \mathrm{~m}, y=0.5 \mathrm{~m}), D(x=0.75 \mathrm{~m}, y=0.25 \mathrm{~m})$ and $E$ $(x=0.75 \mathrm{~m}, y=0.75 \mathrm{~m})$. these feature points are shown in Figure 7.

We obtain the following conclusion from the analysis of Figures 8, 9, 10, 11, and 12.

The displacement time history of each point on membrane surface is superposed by the vibration of each order. The displacement time history of center point $C$ is relatively special. It is a complete and regular cosine curve. This is because the membrane calculated in this computational example is a square; the distances between center point $C$ and each boundary of the membrane are equal, which makes all the higher harmonic waves are counteracted by each other after superposing at center point $C$, leaving a complete and regular cosine wave. We can affirmatively infer that if the membrane is not a square, the displacement and time curve of its center point would not be a complete and regular cosine curve.

\section{Conclusions}

(1) This paper applied L-P perturbation method to solve the governing equations of nonlinear free vibration of rectangular orthotropic membranes in large amplitude, and obtained the approximate analytical solution of the frequency and displacement function of the nonlinear free vibration of rectangular membrane with four edges simply supported.

(2) The comparison and analysis of the computational example proved that using formula (4.21) to calculate the mainly nonlinear free vibration frequency of rectangular orthotropic membranes in large amplitude could satisfy the requirement of engineering precision when the initial displacement is relatively small, and the smaller the initial displacement is, the higher the precision is.

(3) Results obtained from this paper provide some theoretical basis for the calculation and control of nonlinear vibration of membrane structures; and provide some theoretical references for solving the response of membrane structures under dynamic loads and the dynamic design and manufacture of membrane structures.

\section{Acknowledgment}

This paper is supported by the Fundamental Research Funds for the Central Universities (Project no. CDJZR10 2000 10). 


\section{References}

[1] C. Shin, J. Chung, and W. Kim, "Dynamic characteristics of the out-of-plane vibration for an axially moving membrane," Journal of Sound and Vibration, vol. 286, no. 4-5, pp. 1019-1031, 2005.

[2] H. Zhang and J. Shan, "Initial form finding and free vibration properties study of membrane," in Proceedings of the Xi'an International Conference of Architecture and Technology, Proceedings-Architecture in Harmony, pp. 316-320, Xian, China, 2006.

[3] Y. L. Li, C. G. Wang, and H. F. Tan, "Research on free vibration of wrinkled membranes," in Proceedings of the 5th International Conference on Nonlinear Mechanics, pp. 649-654, Shanghai, China, 2007.

[4] J. Pan and M. Gu, "Geometric nonlinear effect to square tensioned membrane's free vibration," Journal of Tongji University, vol. 35, no. 11, pp. 1450-1454, 2007.

[5] S. Yu. Reutskiy, "Vibration analysis of arbitrarily shaped membranes," CMES-Computer Modeling in Engineering \& Sciences, vol. 51, no. 2, pp. 115-142, 2009.

[6] F. Formosa, "Nonlinear dynamics analysis of a membrane Stirling engine: starting and stable operation," Journal of Sound and Vibration, vol. 326, no. 3-5, pp. 794-808, 2009.

[7] P. B. Gonçalves, R. M. Soares, and D. Pamplona, "Nonlinear vibrations of a radially stretched circular hyperelastic membrane," Journal of Sound and Vibration, vol. 327, no. 1-2, pp. 231-248, 2009.

[8] Z. Zhou-Lian, L. Chang-Jiang, H. Xiao-Ting, and C. Shan-Lin, "Free vibration analysis of rectangular orthotropic membranes in large deflection," Mathematical Problems in Engineering, vol. 2009, Article ID $634362,2009$.

[9] X.-T. He and S.-L. Chen, "Perturbation solution to large deflection problem of cantilever beams," Journal of Chongqing Jianzhu University, vol. 25, no. 6, pp. 46-51, 2003.

[10] X.-T. He and S.-L. Chen, "Biparametric perturbation solutions of large deflection problem of cantilever beams," Applied Mathematics and Mechanics, vol. 27, no. 4, pp. 404-410, 2006.

[11] S. T. Mohyud-Din and M. A. Noor, "Homotopy perturbation method for solving fourth-order boundary value problems," Mathematical Problems in Engineering, vol. 2007, Article ID 98602, 15 pages, 2007.

[12] E. Montano, M. Salas, and R. L. Soto, "Nonnegativity preservation under singular values perturbation," Mathematical Problems in Engineering, vol. 2009, Article ID 301582, 25 pages, 2009.

[13] I. V. Andrianov, J. Awrejcewicz, and V. Chernetskyy, "Analysis of natural in-plane vibration of rectangular plates using homotopy perturbation approach," Mathematical Problems in Engineering, vol. 2006, Article ID 20598, 8 pages, 2006.

[14] G. Domairry and A. Aziz, "Approximate analysis of MHD dqueeze flow between two parallel disks with suction or injection by homotopy perturbation method," Mathematical Problems in Engineering, vol. 2009, Article ID 603916, 19 pages, 2009.

[15] F. Shakeri and M. Dehghan, "Solution of delay differential equations via a homotopy perturbation method," Mathematical and Computer Modelling, vol. 48, no. 3-4, pp. 486-498, 2008.

[16] X. Lu and H. -X. Li, "Perturbation theory based robust design under model uncertainty," Journal of Mechanical Design, vol. 131, no. 11, Article ID 111006, 9 pages, 2009.

[17] A. Yildirim, "Application of He's homotopy perturbation method for solving the Cauchy reactiondiffusion problem," Computers and Mathematics with Applications, vol. 57, no. 4, pp. 612-618, 2009.

[18] Z. Z. Ganji, D. D. Ganji, H. Jafari, and M. Rostamian, "Application of the homotopy perturbation method to coupled system of partial differential equations with time fractional derivatives," Topological Methods in Nonlinear Analysis, vol. 31, no. 2, pp. 341-348, 2008.

[19] E. L. Jansen, "A perturbation method for nonlinear vibrations of imperfect structures: application to cylindrical shell vibrations," International Journal of Solids and Structures, vol. 45, no. 3-4, pp. 1124-1145, 2008. 


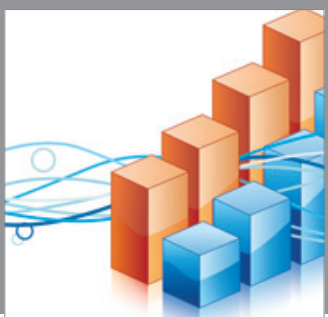

Advances in

Operations Research

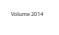

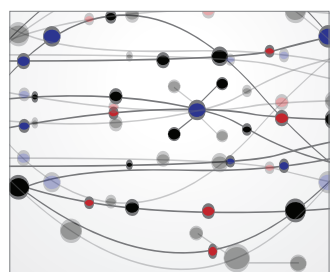

\section{The Scientific} World Journal
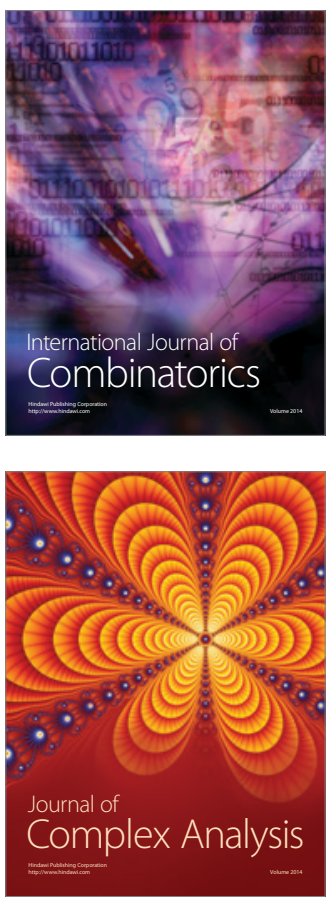

International Journal of

Mathematics and

Mathematical

Sciences
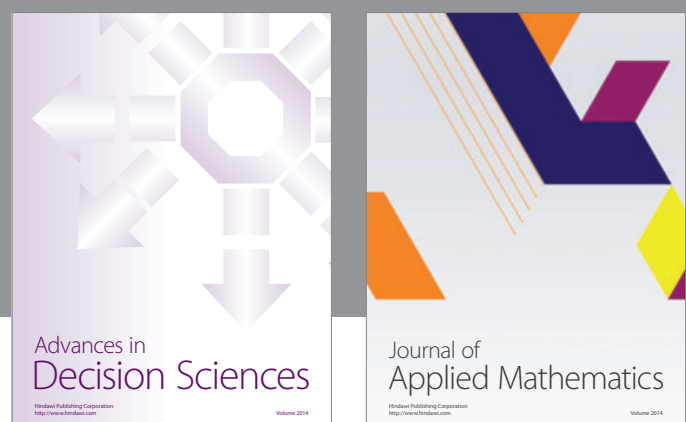

Journal of

Applied Mathematics
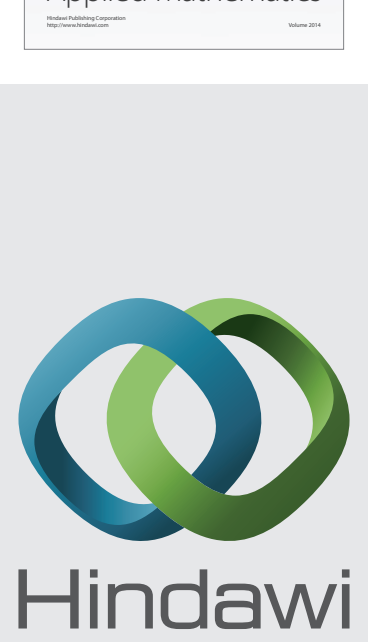

Submit your manuscripts at http://www.hindawi.com
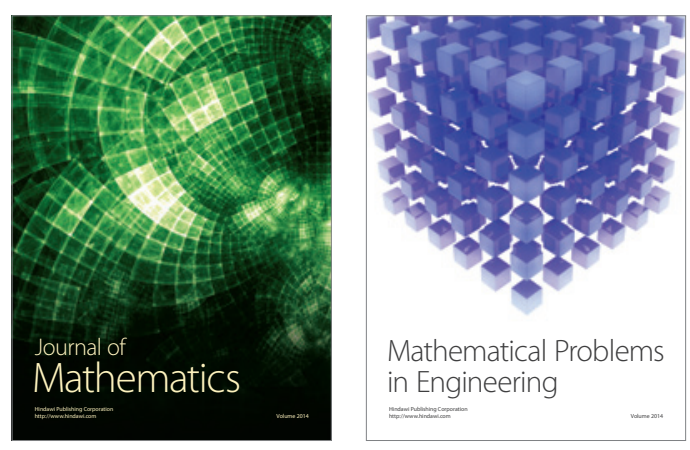

Mathematical Problems in Engineering
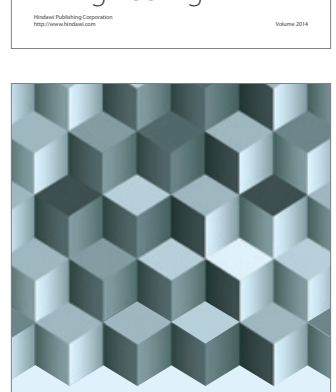

Journal of

Function Spaces
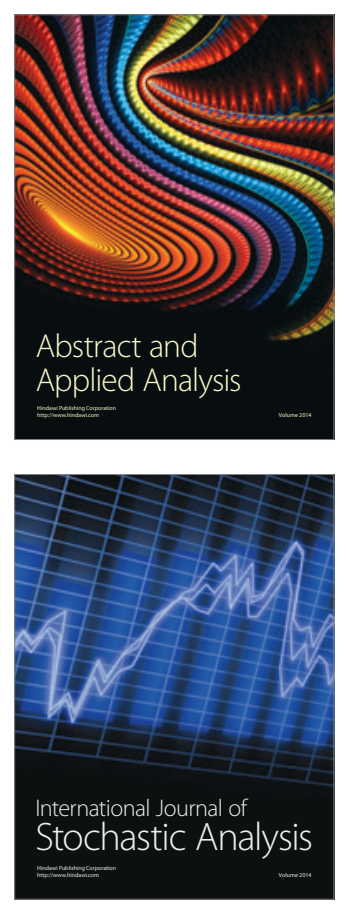

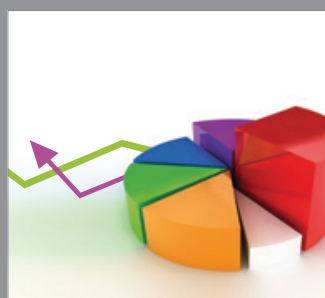

ournal of

Probability and Statistics

Promensencen
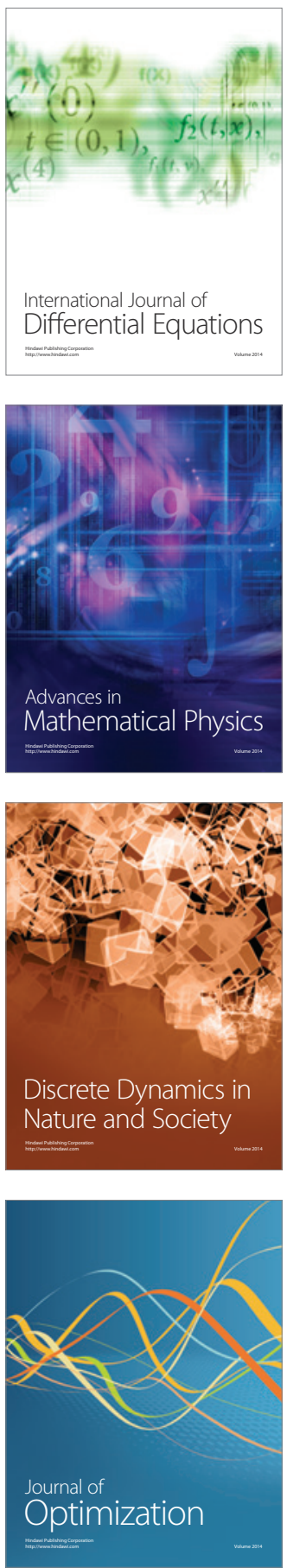